\title{
The Effectiveness of the Spiritual-Islamic Healing Group Therapy on Increasing the Resilience and Life Hope of Veterans with Mental Disorders
}

\section{ART ICLE INF O}

\section{Article Type}

Original Research

\section{Authors}

Afshari A*1 $P h D$

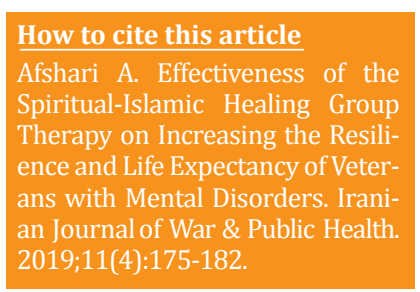

${ }^{1}$ Psychology Department, Human Science Faculty, University of Maragheh, Maragheh, Iran

\section{*Correspondence}

Address: University of Maragheh, Madar Square, Amir Kabir Highway, Maragheh, East Azerbaijan, Iran. Postal code: 5518183111 Phone: +98 (41) 37276008 Fax: -

a_afshari@maragheh.ac.ir

\section{Article History}

Received: February 12, 2019

Accepted: June 15, 2019

ePublished: December 21, 2019

\section{A B S T R A C T}

Aim(s) The war and its resulting stresses caused mental disorders as a prevalent problem in disabled veterans and their families. The aim of this study was to investigate the effectiveness of the spiritual-Islamic healing group therapy on increasing the resilience and life expectancy of veterans with mental disorders.

Instruments \& Methods This semi-experimental study with the pretest-posttest design was carried out on 30 people of veterans with mental disorders in Maragheh city in 2018. The subjects were selected by convenience sampling method and randomly assigned in to the two control and experimental groups ( $n=15$ in each group). The research instruments were the Connor-Davidson resilience scale and Schneider life expectancy scale. The spiritualIslamic healing group therapy was implemented for the experimental group in 8 sessionsin 90 minutes during the 10 weeks. Data were analyzed through SPSS 21 software using multivariable analysis of covariance test (MANCOVA).

Findings By controlling the effect of the pretest, there was a significant difference between the mean scores of the posttest stage in the life expectancy and resilience in the control and experimental groups. After the intervention, the mean scores of these variables in the experimental group was higher than the control group $(\mathrm{p}<0.05)$.

Conclusion The spiritual-Islamic healing group therapy improves the resilience and life expectancy in veterans with mental disorders.

Keywords Spiritual-Islamic Healing Group; Resilience; Life Expectancy; Veterans

\section{I T A T I O N L I N KS}

[1] Comparison the effect of training of conflict ... [2] DoD telemental health ... [3] VA Puget Sound telemental health service to rural ... [4] The comparison between mental health of devotee's spouses ... [5] Qualification of studies conducted on ... [6] Viewpoints of veterans affected by ... [7] Role of secondary post-traumatic ... [8] Internal consistency and confirmatory ... [9] Development of a new resilience ... [10] Resilience in ecosystemic context ... [11] Resilient children are less tests ... [12] Self-compassion, interpersonal ... [13] Male student veterans: Hardiness, psychological ... [14] The self-compassion scale is a valid and ... [15] Stress, resilience and leisure ... [16] Affective mediators of the influence of ... [17] The role of resilience and ... [18] Resilience and indicators of adjustment ... [19] Assessment of existential meaning ... [20] Dynamics of hope in adults living with ... [21] Meaning in life and hope as predictors ... [22] The role of hope as a mediator ... [23] Insight into severe mental illness ... [24] The relationship of hope ... [25] Subjective quality of life in patients ... [26] Recovery components as determinants ... [27] Positive psychology progress: empirical ... [28] The role of hope in cognitive behavior ... [29] The effect of spiritual intelligence training ... [30] the effectiveness of the spiritual ... [31] The relationship of spiritual intelligence ... [32] Post-traumatic stress disorder, social ... [33] Spirituality and distress in palliative ... [34] Teachers' role in elementary school mental ... [35] A Spiritual Strategy for Counseling ... [36] Relationship between anxiety and ... [37] Casebook for a spiritual strategy in counseling ... [38] The conservative church: Psychological ... [39] Resilience factors in individuals at risk for ... [40] Effects of resilience on mental ... [41] Hope and academic success in ... [42] Academic procrastination and the ... [43] Psychometric features of Omid ... [44] The relationship between life's meaningfulness ... [45] A facilitator's manual for use with ... [46] The effectiveness of group based ... [47] The effectiveness of group spiritual ... [48] Use of Tai Chi to treat mental health disorders ... [49] Purpose, hope, and Life Satisfaction ... [50] Hope as a source of resilience in later... 
استرس يّ از حادثه، اختلالات خلقى و اضطرابى، يرخاشكرى،

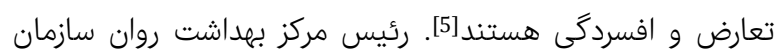
بنياد شهيد و امور ايثاركران، تعداد جانبازان اعصاب و و روان كشئرور

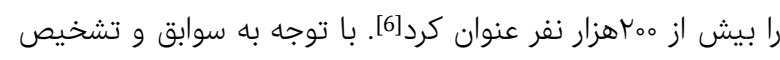

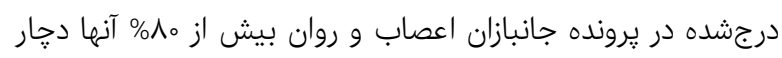

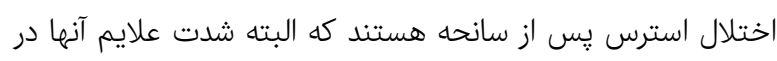

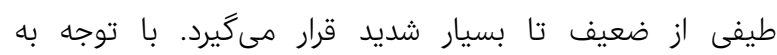

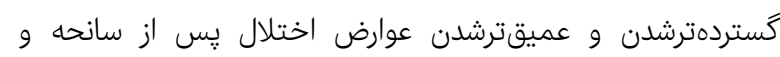

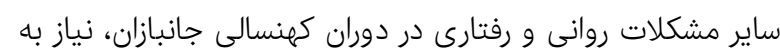

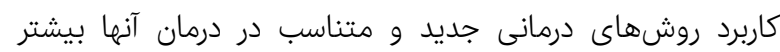

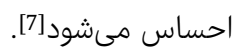

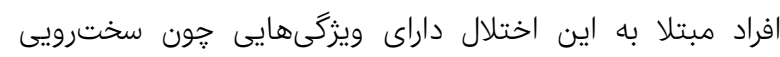

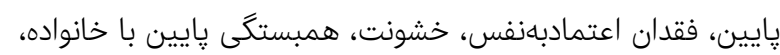

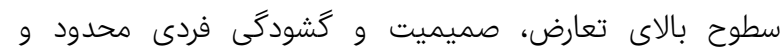

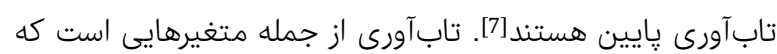

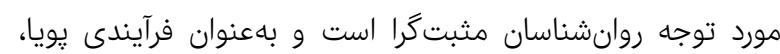

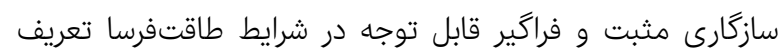

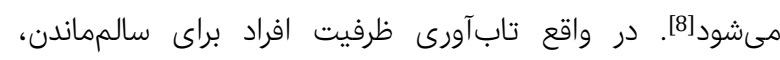

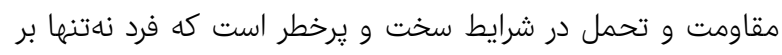

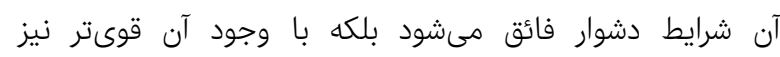

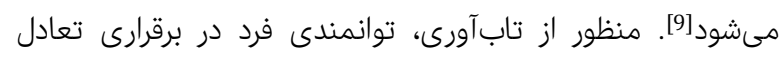

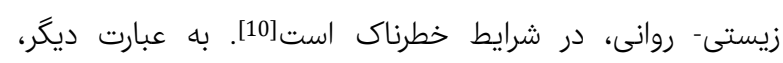

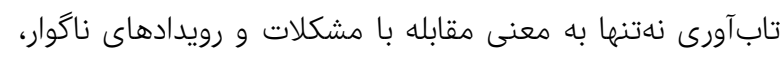

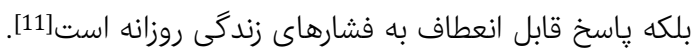

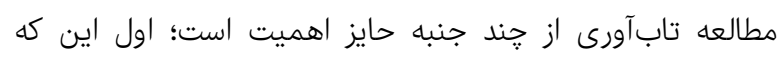

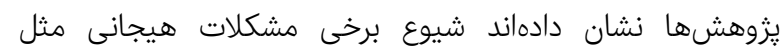

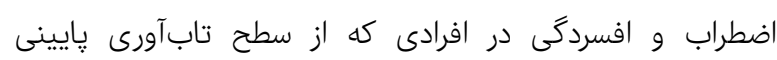

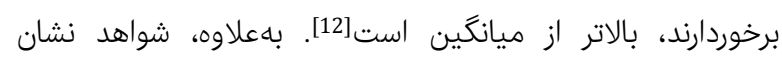

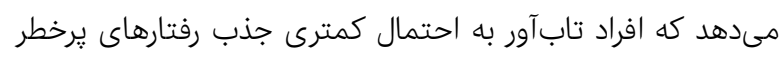

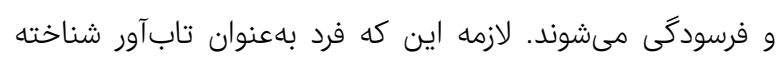

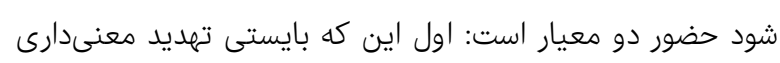

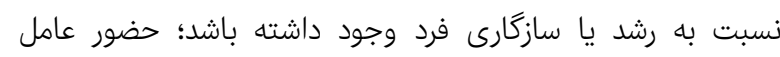

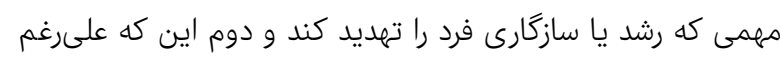

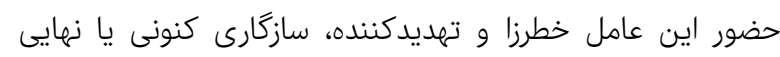

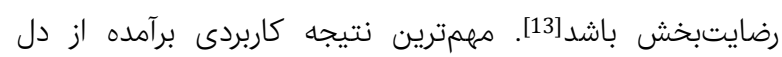

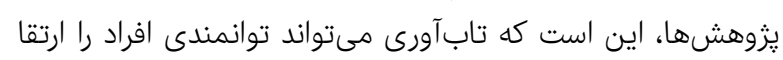

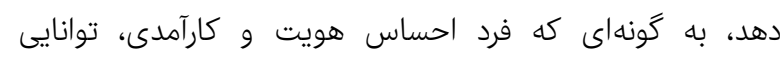

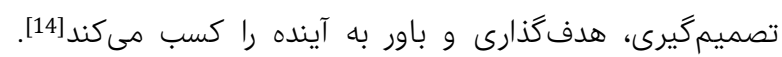

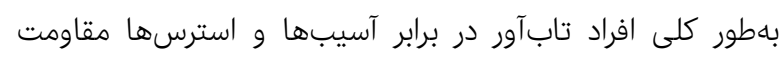

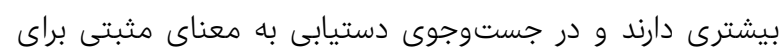

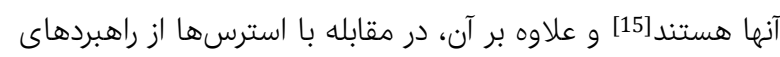

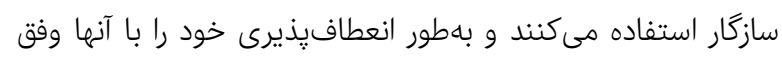

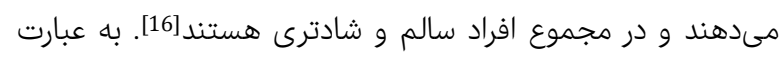

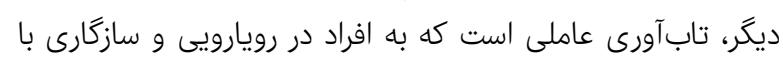

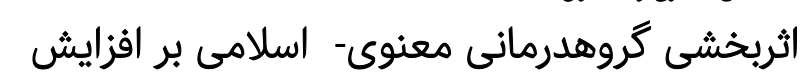

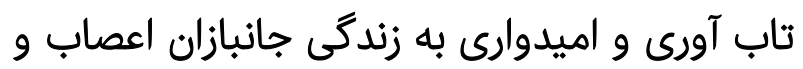
روان

كَروه روانشناسى، دانشكده علوم انسانى، دانشكاه مراغه، مراغه، ايران

جكيده

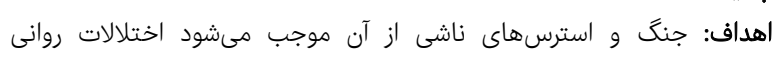

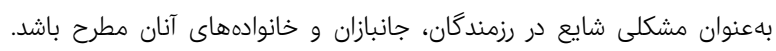

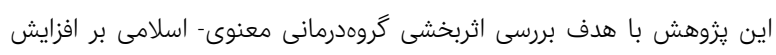

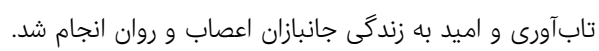

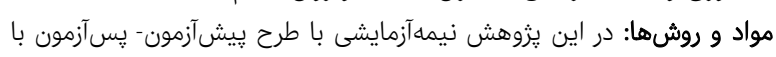

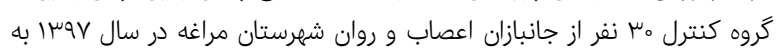

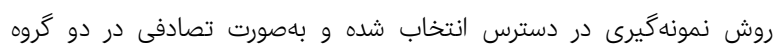

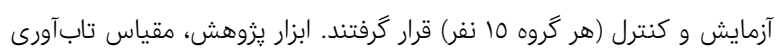

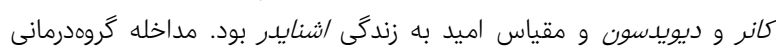

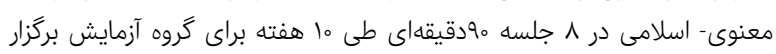

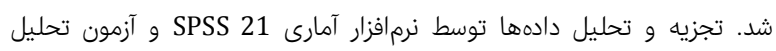

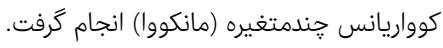

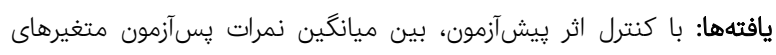

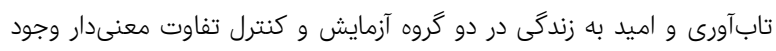

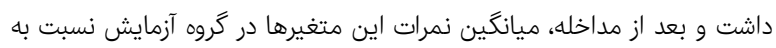

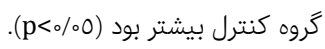

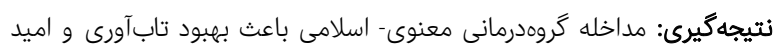

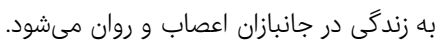

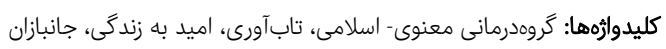

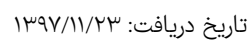

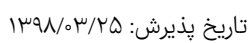
a_afshari@maragheh.ac.ir :نويسنده مسئول: بذيرش:

مقدمه

جنگ بهعنوان ضربهاى روانشناختى است كه خارج از تجربيات

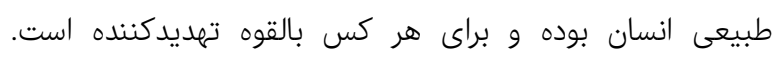

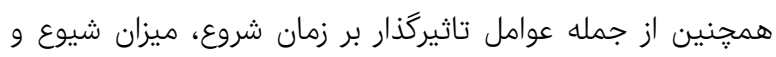

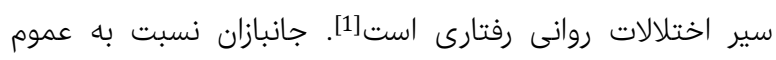

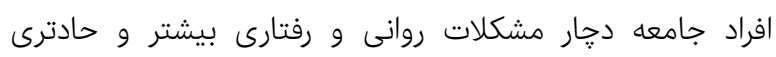

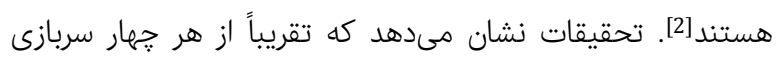

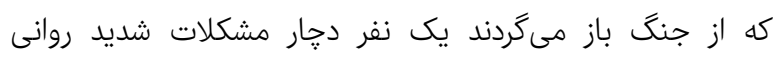

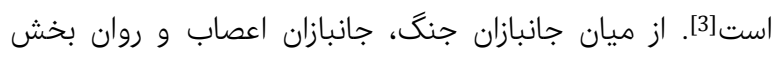

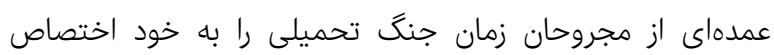

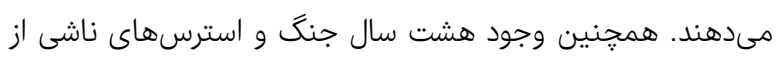

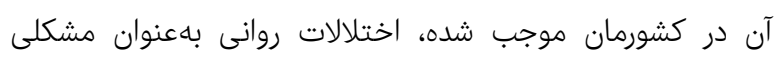

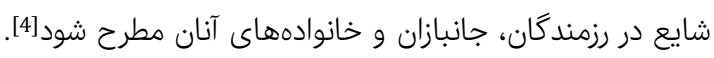

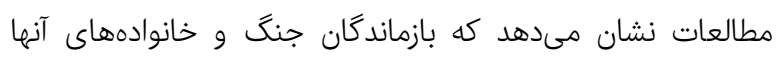
مبتلا به طيف وسيعى از آسيبهاى روانى همجيون اختان خانلال 


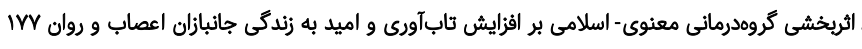

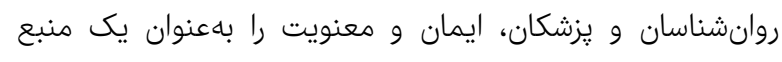

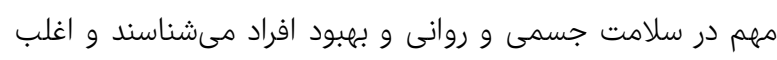

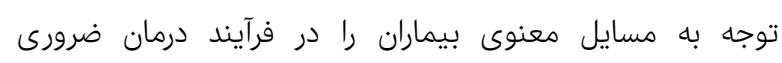

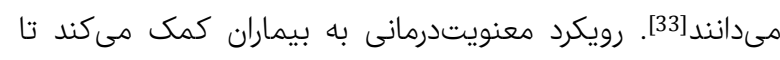

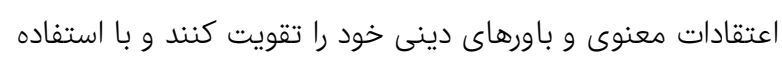

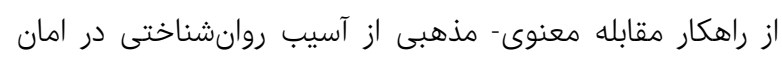

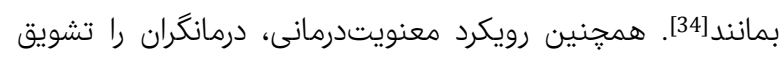

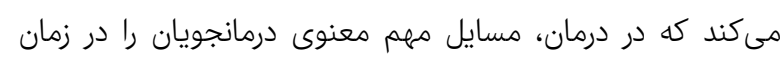

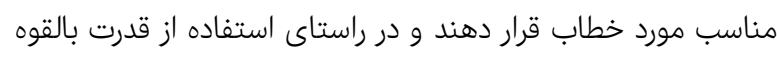

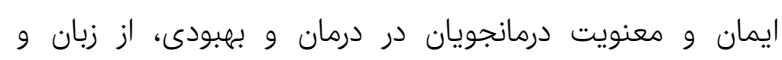

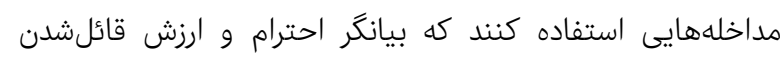

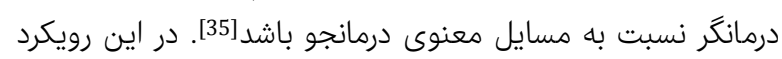

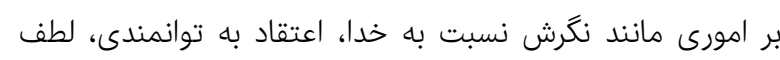

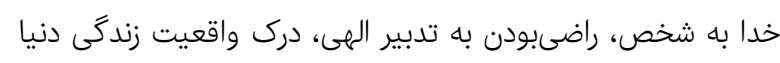

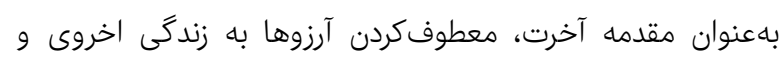

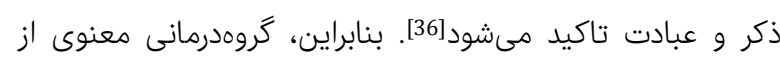

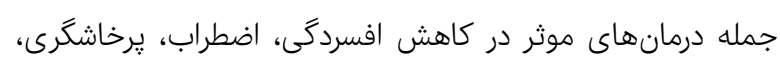

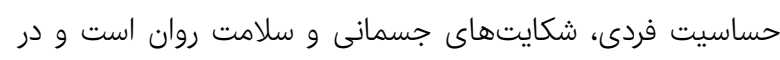

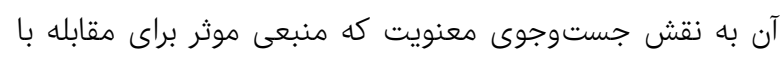

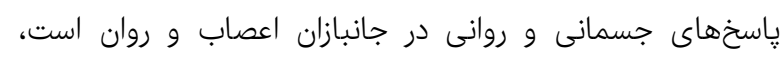

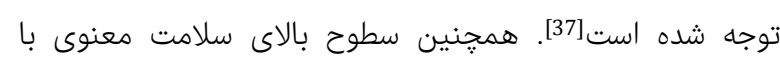

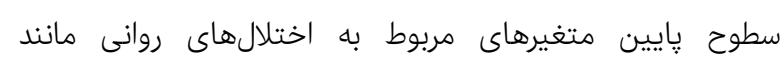

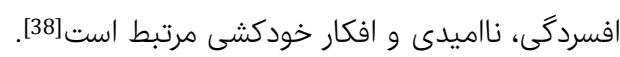

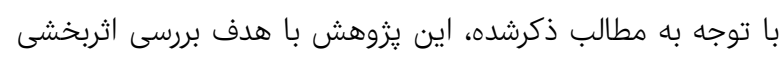

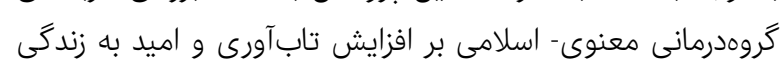

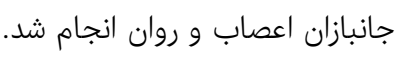

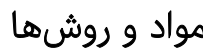

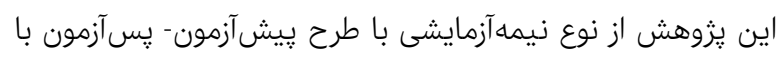

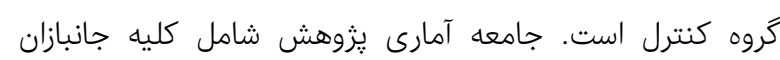

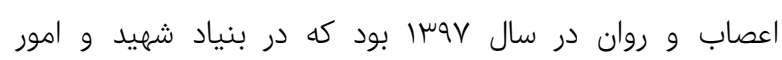

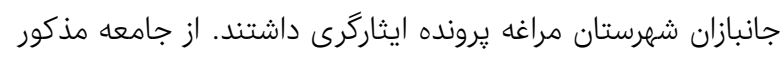

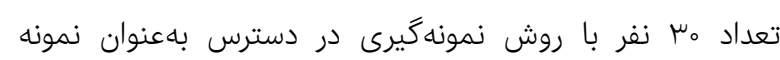

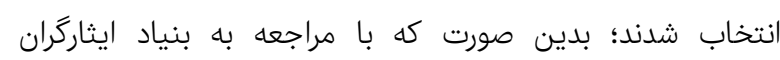

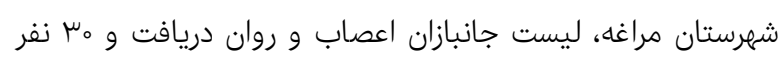

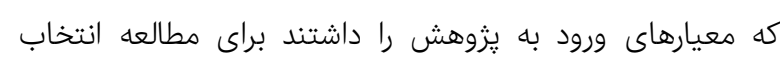

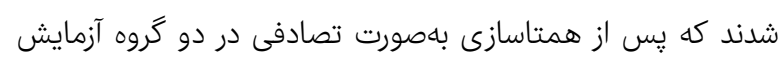

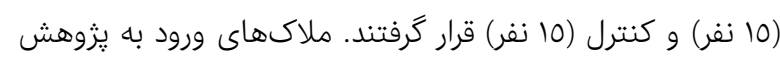

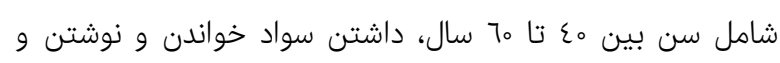

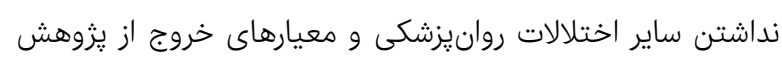

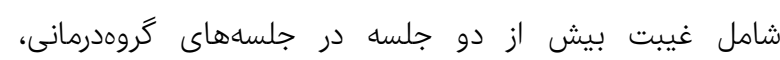

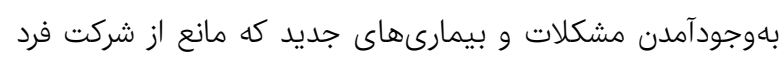

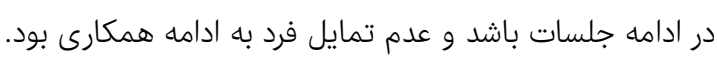

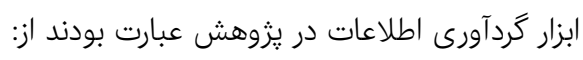

موقعيتهاى سخت و تنشزاى زندگى كمك مى كند، افسردگى را

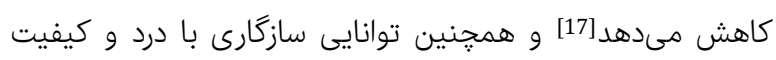

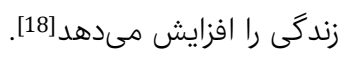

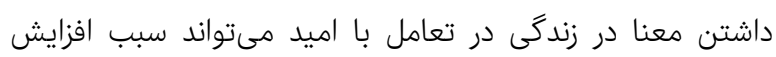

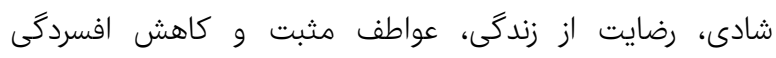

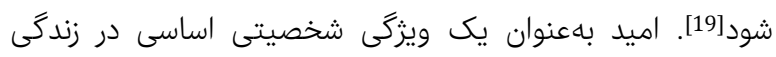

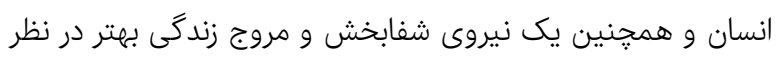

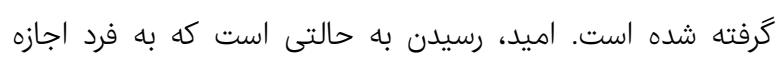

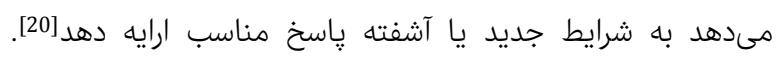

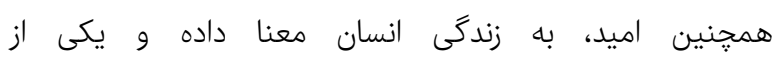

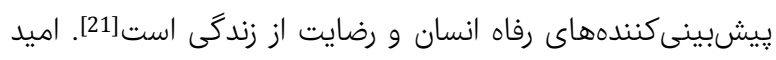

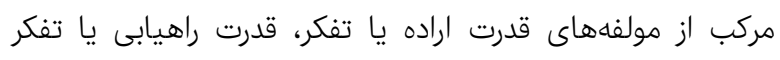

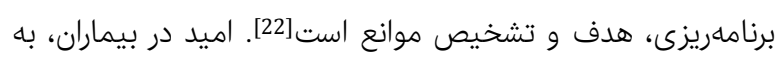

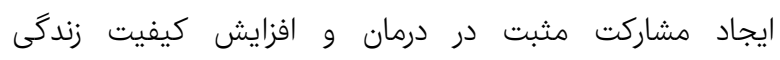

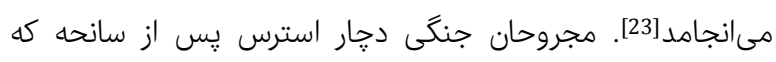

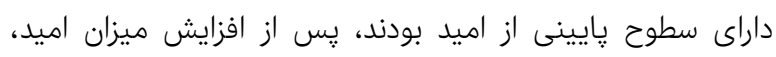

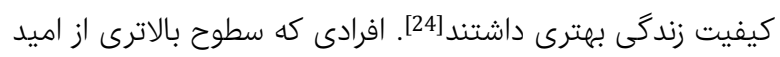

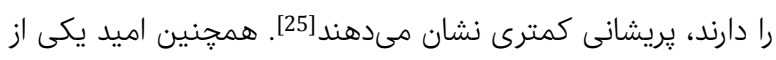

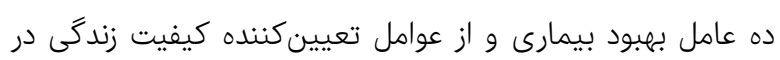

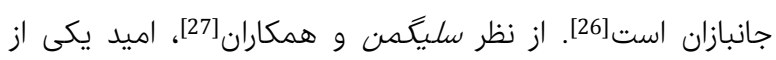

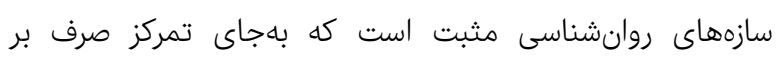

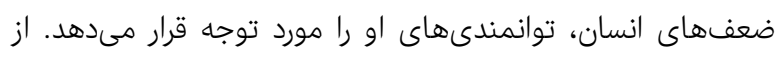

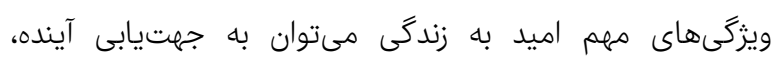

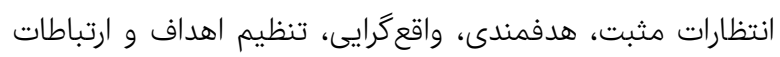

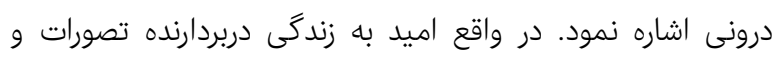

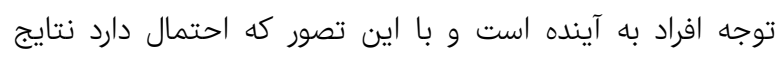

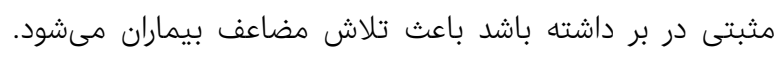

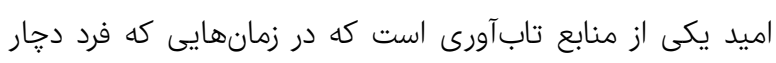

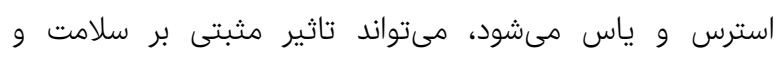

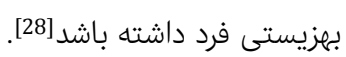

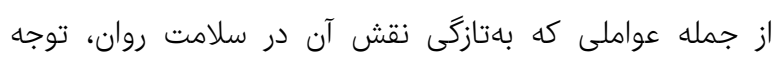

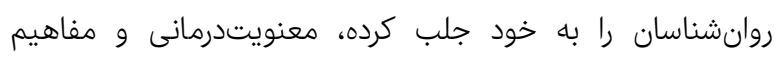

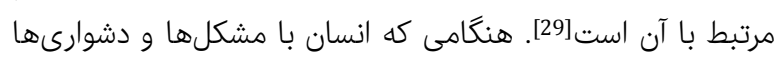

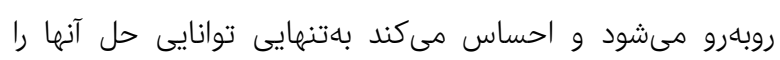

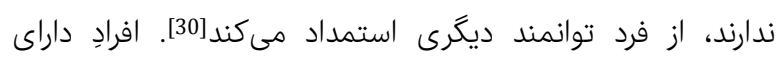

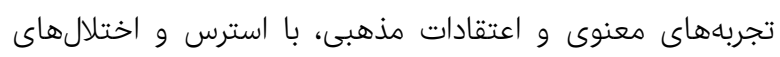

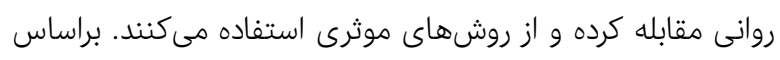

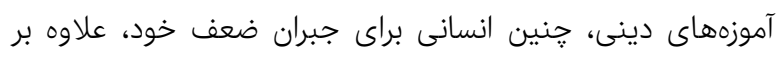

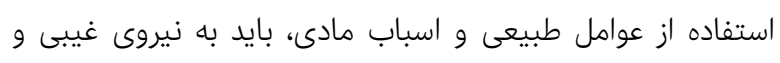

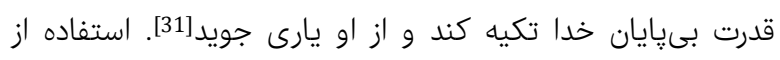

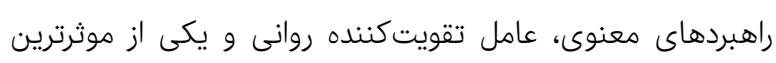

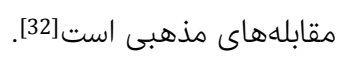

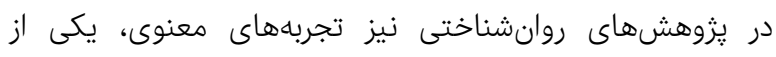

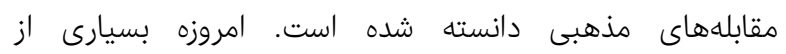


كردند. كَروه كنترل در اين مدت هيج گَونه مداخلهاى دريافت نكرد.

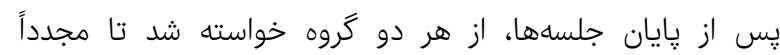

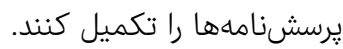

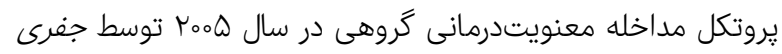

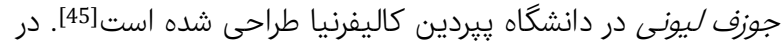

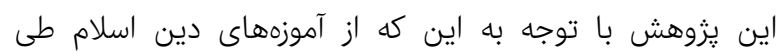

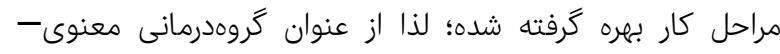
اسلامى استفاده شد. براى تعيين روايى تروتئه

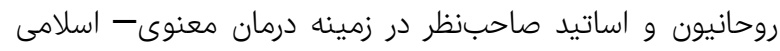

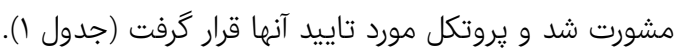

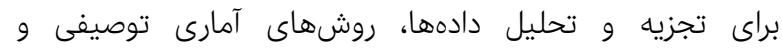

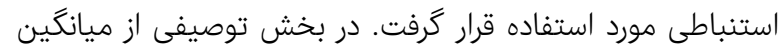

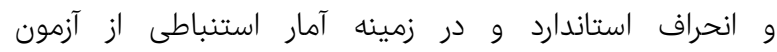

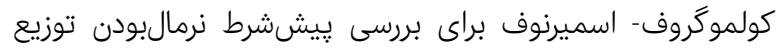

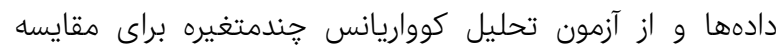

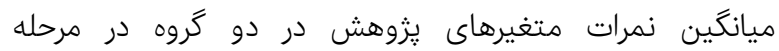

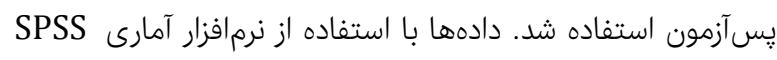
21 تحليل شدند.

جدول () يروتكل گروهدرمانى معنوى- اسلامى إمل آشنايى اعضا با يكديكر و گفتو گو در مورد معنويت و دين و تاثير آن در زندگى

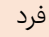
جلسه دوم خودآكاهى و ارتباط با خود و گوشدادن به نداى درونى، آموزش حل مساله و اصلاح رفتار

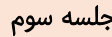

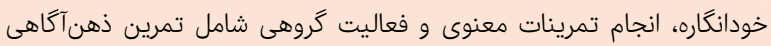

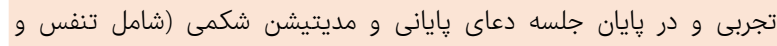

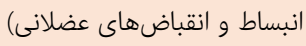

جلسه جهارم كلمه خدا، ارتباط با خدا و اعتقاد به عدالت خدايى جلسه ينجم طلب عفو و بخشش و در آخر جلسه مراقبه جلسه ششم

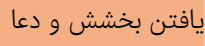
جلسه هفتم شناخت و توجه به ارزشها (جه جيزى از لحاظ اخلاقى درست است كه انجام دهيم؟)

جلسه هشتم ساختن معنا، معنادار ساختن زندگى مشتى

\section{يافتهها}

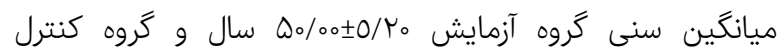

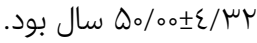

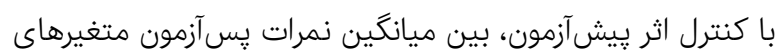

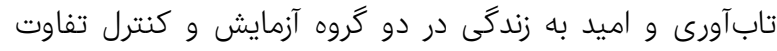

ا- مقياس تابآورى: بهمنظور سنجش تابآورى جانبازان از

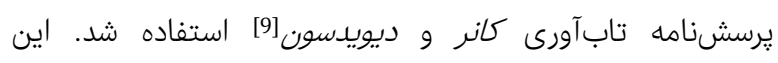

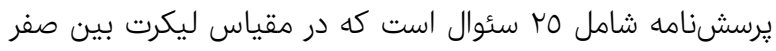

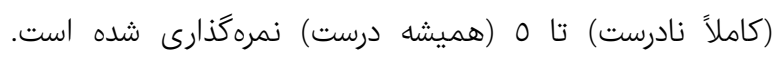

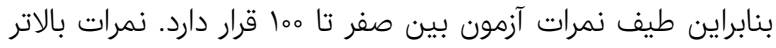

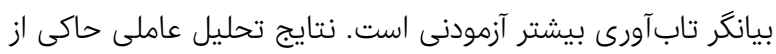

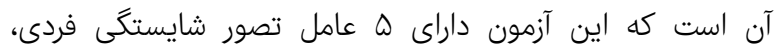

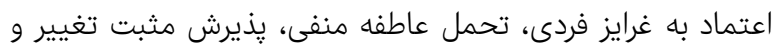

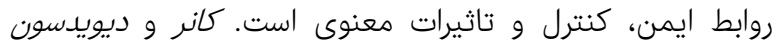

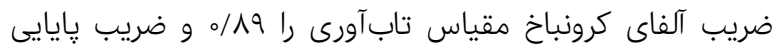

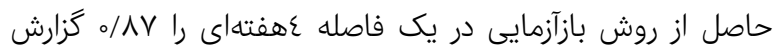

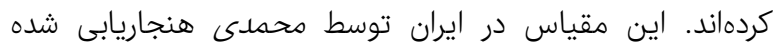

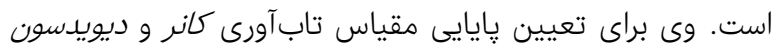

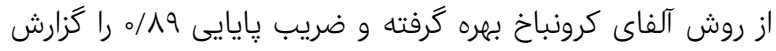

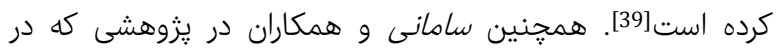

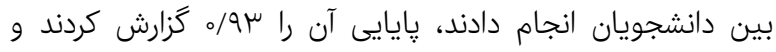

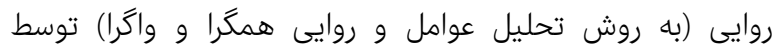

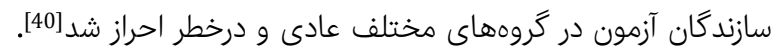

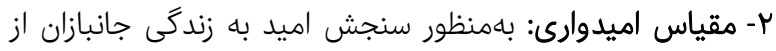

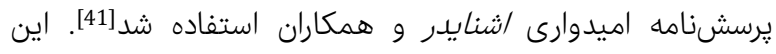

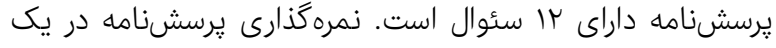

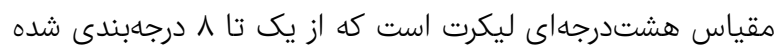

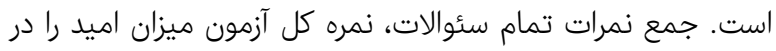

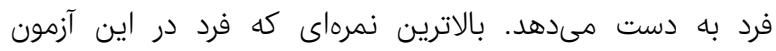

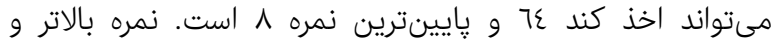

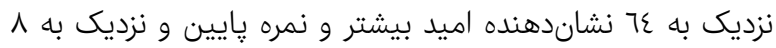

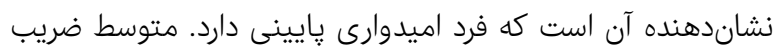

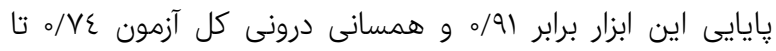

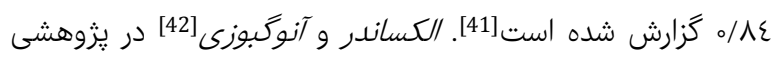

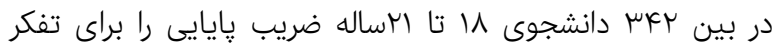

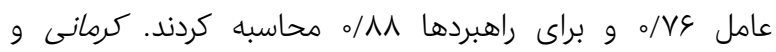

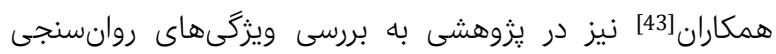

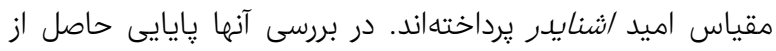

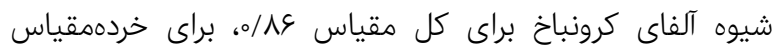

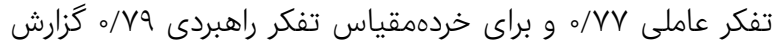

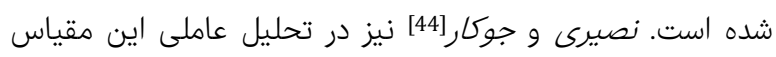

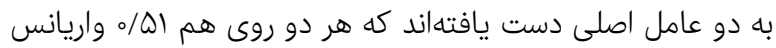
اميد را تبيين مىكردند. يس از تعيين نمونه يزوهشى، توضيحات لازم درباره اهداف

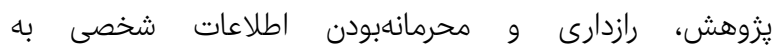

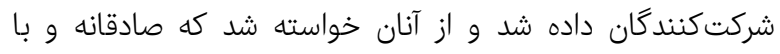

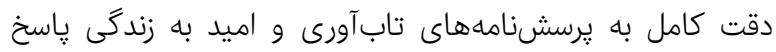

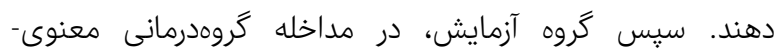
اسلامى كه در ^ جلسه مودقيقهاى طى ما هفته بركزار شد، شركت 


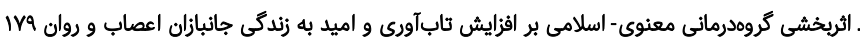

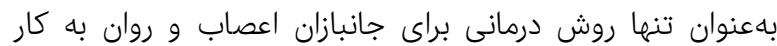

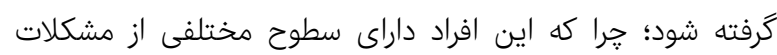

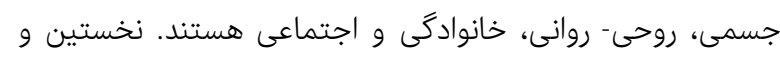

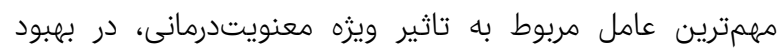

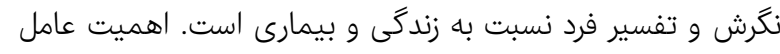

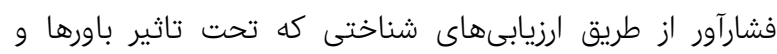

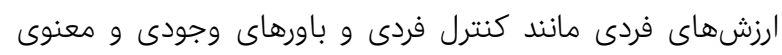

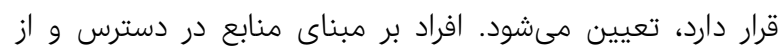

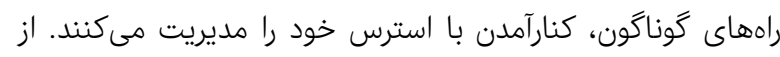

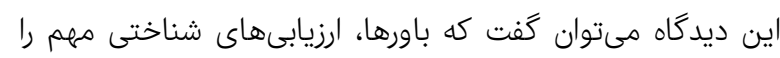

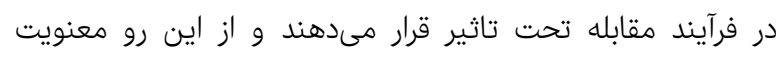

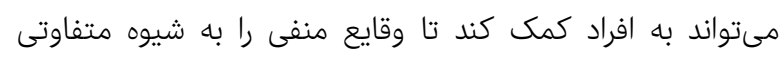

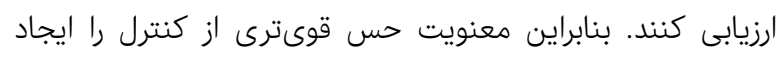

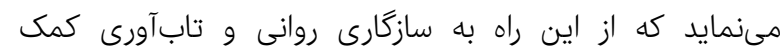

مى كند [42].

يافتههاى يزوهش حاضر نشان داد كه اميد به زندگى جانبازانى كه

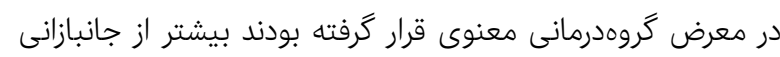

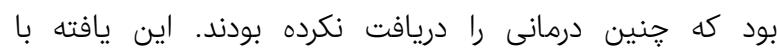

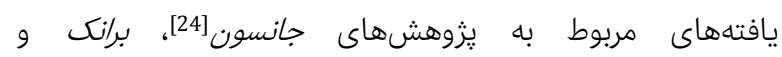

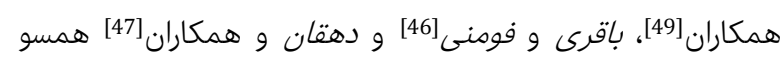

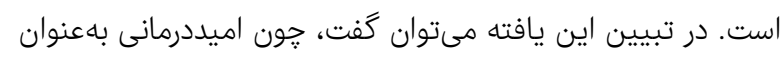

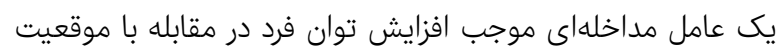

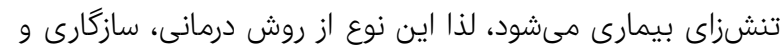

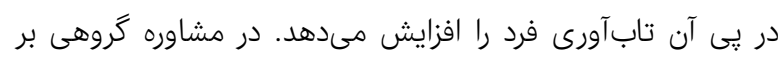

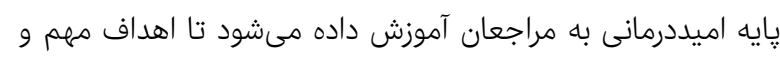

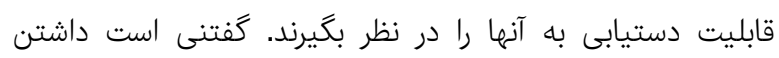

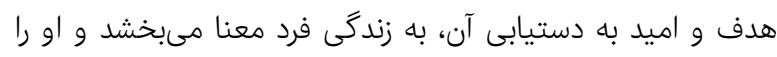

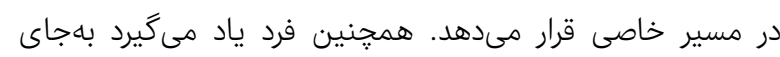

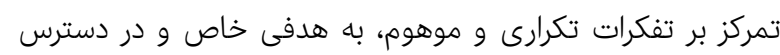

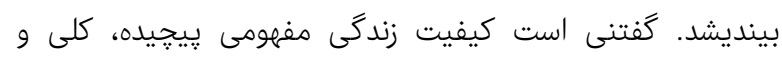
קندجانبه است كه علاوه بر برداشت ذهنى فرد، به شرايط جسمانى

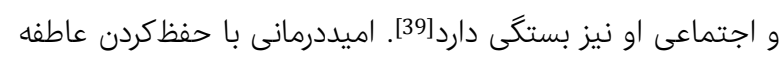

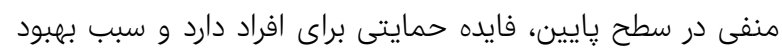

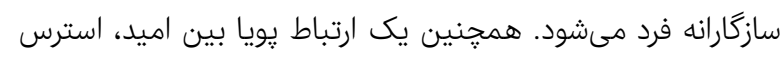

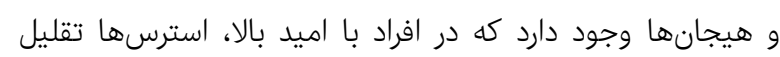

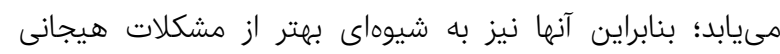

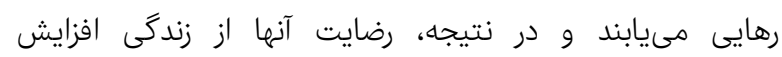

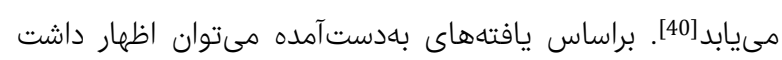

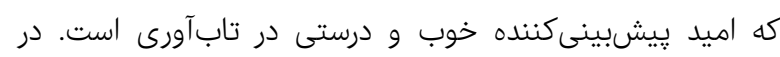

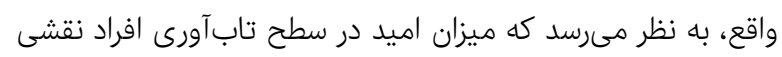

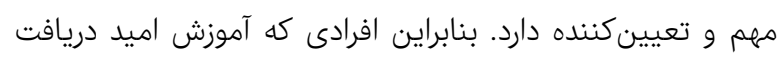

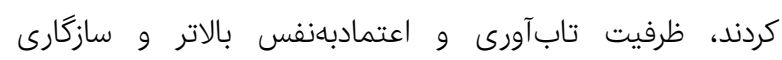

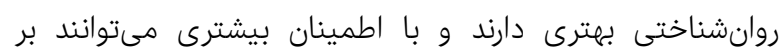

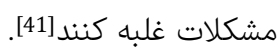

معنىدار وجود داشت و بعد از مداخله، ميانگين نمرات اين

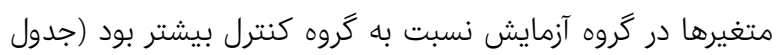

جدول r r) مقايسه ميانكين آمارى نمرات متغيرهاى يزوهش در در مراحل

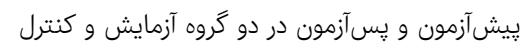

\begin{tabular}{|c|c|c|c|c|c|}
\hline مجذور & معنىدارى سطح & مقدار & ״سآزمون & يیش آزمون & متغيرها \\
\hline D & $\%$ \% & $r / v q$ & $\begin{array}{l}00 / 00 \pm r / R r \\
0 V / r T \pm r / V\end{array}$ & $\begin{array}{l}\varepsilon q / r_{0} \pm r / r_{\Lambda} \\
\varepsilon q / \circ\rceil \pm r / q \Lambda\end{array}$ & ترابآورى كروه \\
\hline o/KrA & $\% \mu$ & $1 / 07$ & $\begin{array}{l}\varepsilon \circ / \Lambda_{0 \pm} \pm / O r \\
\varepsilon T / / \mu_{ \pm} /{ }^{\mu}\end{array}$ & $\begin{array}{l}\varepsilon \circ / V \gamma_{ \pm} \pm r / \mu V \\
\varepsilon 1 / \circ\rceil \pm r / \varepsilon \Lambda\end{array}$ & 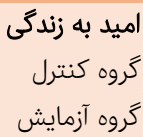 \\
\hline
\end{tabular}

بحث

يزوهش حاضر با هدف بررسى اثربخشى گروهدرمانى معنوى-

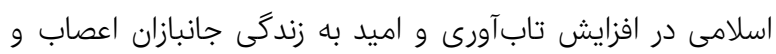

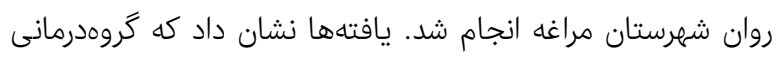

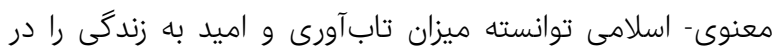

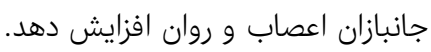

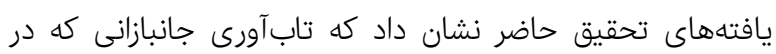

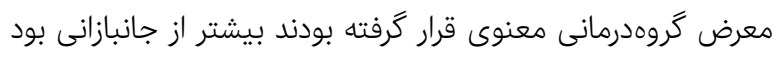

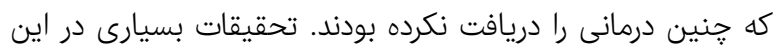

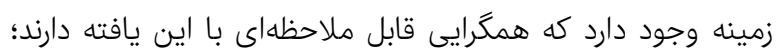

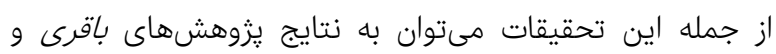

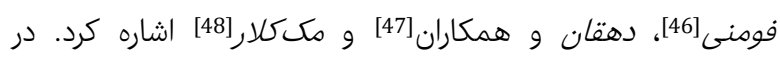

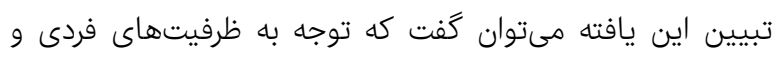

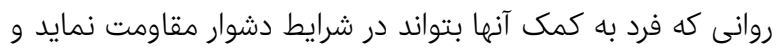
دجار آسيبديدگى نشود و حتى در تجربه شرايط مشكل آفرين يا

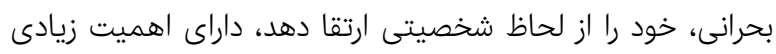

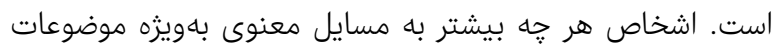

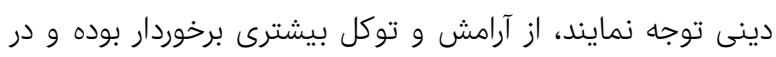

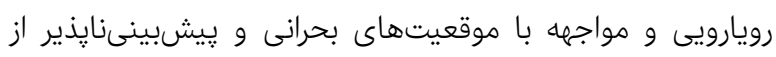

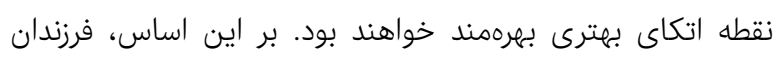

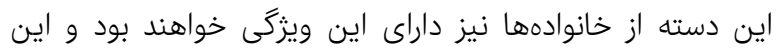

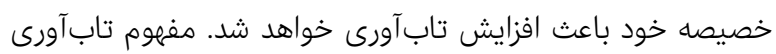

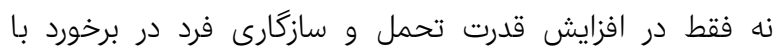

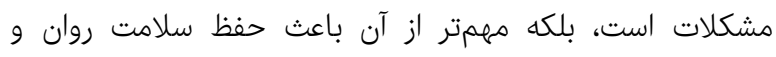

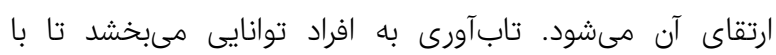

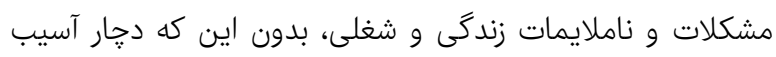

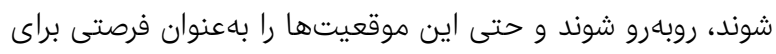
ارتقا و رشد شخصيت خود مورد استفاده قرار دهند.

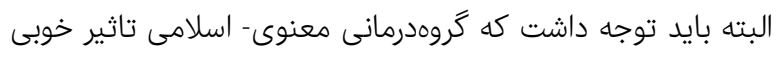

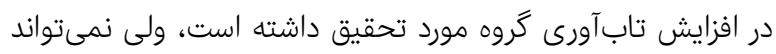


به زندگى در جانبازان اعصاب و روان مىشود.

تشكر و قدردانى: از مسئولان بنياد شهيد و امور ايثاركران شهرستان

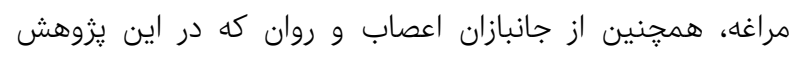

شركت داشتند صميمانه قدردانى مى آىود.

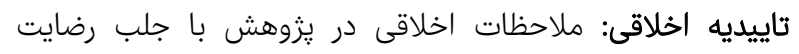

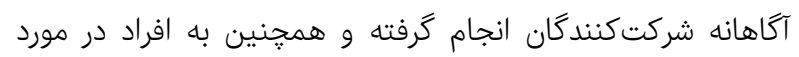

محرمانهبودن نتايج اطمينان داده شده است.

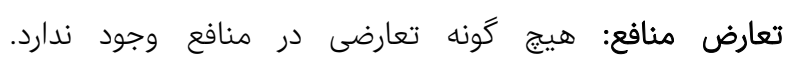
منابع مالى: اين مطالعه از سوى هيج سازمانى مورد حمايت مالى قرار نكَرفته است.

منابع

1- Parandeh A, Sirati Nir M, Khaghanizadeh M, Karimi Zarchi AA. Comparison the effect of training of conflict resolution and relaxation on quality of life's spouses of war veterans affected posttraumatic stress disorder with major depression disorder. Q J Fundam Ment Health. 2007;9(33-34):67-76. [Persian]

2- Kramer GY, Ayers T, Mishkind M, Norem A. DoD telemental health guidebook. Arlington County: Defense Centers of Excellence; 2011.

3- Smucker Barnwell SV, Juretic MA, Hoerster KD, Van de Plasch R, Felker BL. VA Puget Sound telemental health service to rural veterans: a growing program. Psychol Serv. 2012;9(2):209-11.

4- Vafaei T, Khosravi S. The comparison between mental health of devotee's spouses and normal person's spouses. Iran J War Public Health. 2009;1(4):9-13. [Persian]

5- Ahmadi K, Shahi R, Habibi M. Qualification of studies conducted on the mental health status of veterans: a systematic review. J Behav Sci. 2011; 5(3):217-24. [Persian]

6- Ahmadi M, Bahaadinbeigi K, Noori T. Viewpoints of veterans affected by psychological disorders towards usage of telemental services for war veterans. Iran J War Public Health. 2013;6(1) :51-9. [Persian]

7- Rezapour Mirsaleh Y, Behjatmanesh A, Tavallaei SV. Role of secondary post-traumatic stress disorder and resilience on academic motivation and performance of veterans' student children. Iran J War Public Health. 2016:8(4):225-33. [Persian]

8- Keihani M, Taghvaei D, Rajabi A, Amirpour B. Internal consistency and confirmatory factor analysis of the Connor-Davidson resilience scale (CD-RISC) among nursing female. Iran J Med Educ. 2015;14(10):857-65. [Persian]

9- Conner KM, Davidson JRT. Development of a new resilience scale: The Conner-Davidson Resilience Scale (CD-RISC). Depress Anxiety. 2003; 18(2): 76-82.

10- Waller MA. Resilience in ecosystemic context: evolution of the child. Am J Orthopsychiatry. 2001;71(3):290-7.

11- Putwain DW, Nicholson LJ, Connors L, Woods K. Resilient children are less tests anxious and perform better in tests at the end of primary schooling. Learn Individ Differ. 2013;28(2):41-6.

12- Yarnell LM, Neff KD. Self-compassion, interpersonal conflict resolutions, and well-being. Self Identity.
بهطور كلى درمان معنوى يك برنامه درمانى است كه بهارن بهنظور

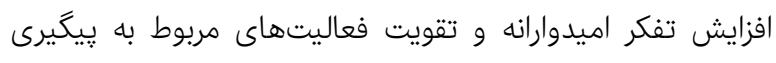

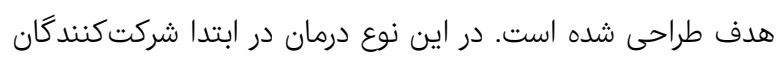

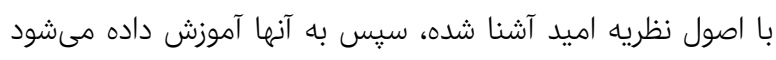

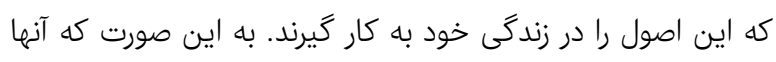

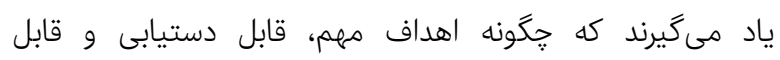

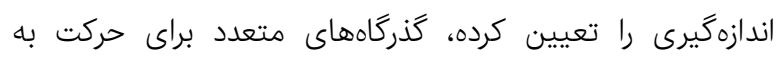

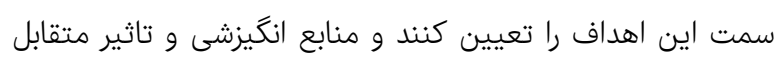

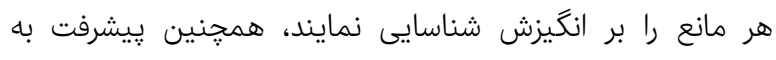

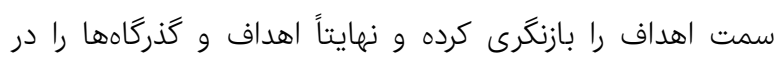

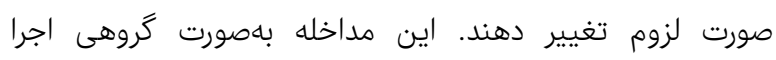

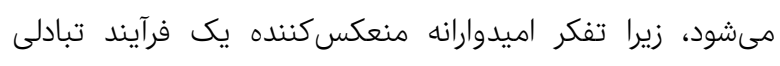

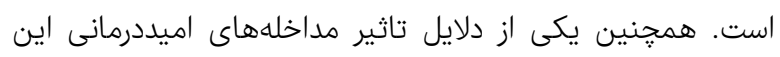

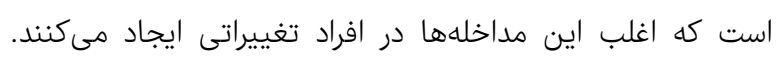

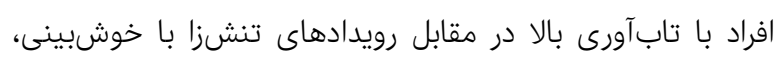
ابراز وجود و اعتمادبهنفس برخورد مىكنند؛ در نتيجه اين رويدادها باديا

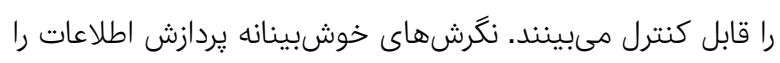

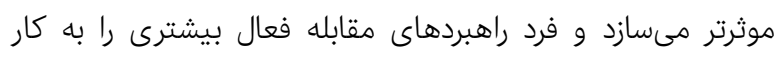

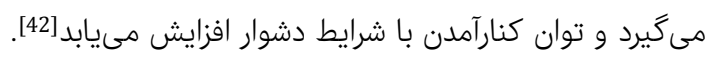

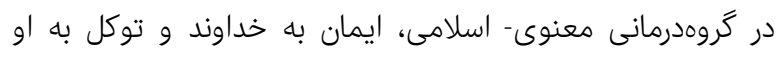

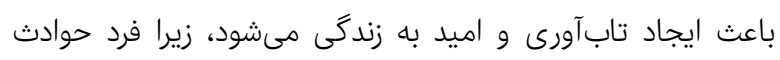

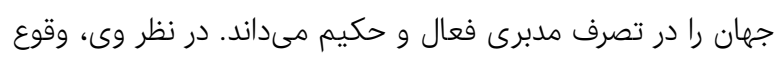
حوادث جهان براساس حكمت است، جهان هستى واجد نظم بوده

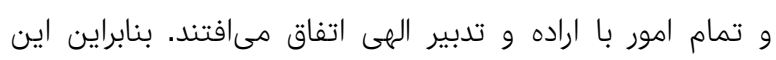
ديدكاه كه حوادث جهان تصادفى نيست و تحت تدبير و قدرت

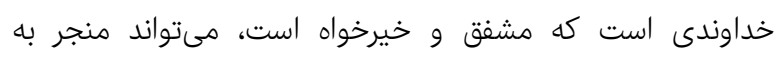

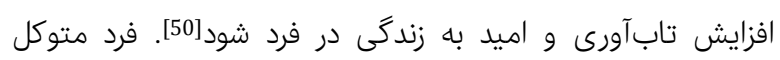

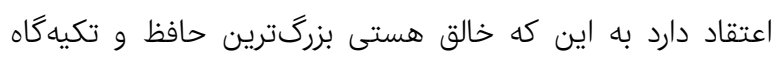

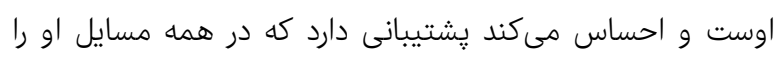

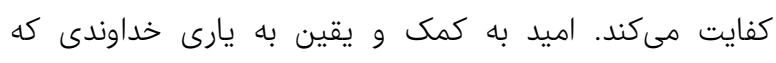
قدرتش فوق قدرتهاست، باعث مىشود كه فرد به اين اطمينان برسد كه با يارى مدبر هستى، توانايى مقابله با مشكلات رات را دارد.

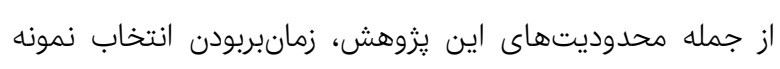

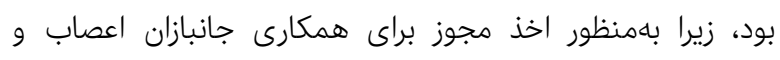

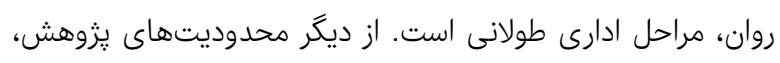

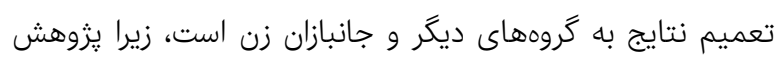
روى نمونه مردان انجام شده است.

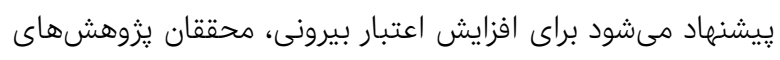

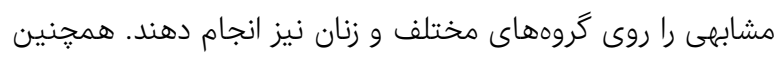

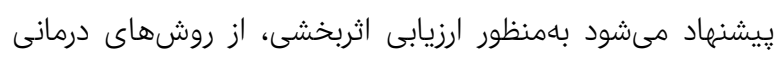
فردمحور نيز استفاده شود.

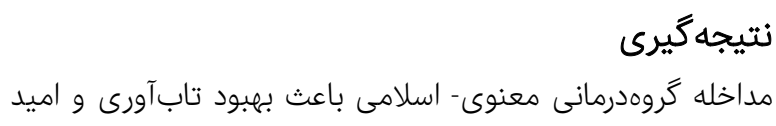




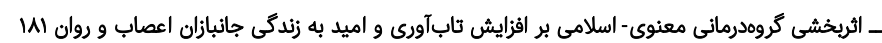
Mousavi MR. the effectiveness of the spiritual treatment groupon improving the quality of life and mental health in women with breast cancer. J Res Relig Health. 2017;3(1):64-78. [Persian]

31- Entesar Foumany G, Daneshdoost M. The relationship of spiritual intelligence with mental health and organizational commitment among nurses in Mashhad Hospitals. J Edu Manag Studi. 2014;3(4):36-9. [Persian]

32- Kashdan TB, Morina N, Priebe S. Post-traumatic stress disorder, social anxiety disorder, and depression in survivors of the Kosovo War: Experiential avoidance as a contributor to distress and quality of life. J Anxiety Disord. 2009;23(2):185-96.

33- Hills J, Paice JA, Cameron JR, Shott S. Spirituality and distress in palliative care consultation. J Palliat Med. 2005;8(4):782-8.

34- Kaveh MH, Shojaeizadeh D, Shahmohammadi D, Eftekhar Ardebili H, Rahimi A, Bolhari J. Teachers' role in elementary school mental health: Results of an interventional study. J Payesh. 2003;2(2):101-9. [Persian]

35- Richards PS, Bergin AE. A Spiritual Strategy for Counseling and Psychotherapy. $2^{\text {nd }}$ Edition. Washington D.C.: American Psychological Association; 2005.

36- Ghobari Bonab B, Motevallipour A, Hakimi Rad E, Habibi Asgarabad M. Relationship between anxiety and depression with spirituality in Tehran University Students. J Appl Psychol. 2009;3(2):110-23. [Persian] 37- Richards PS, Bergin AE. Casebook for a spiritual strategy in counseling and psychotherapy. . Washington D.C.: American Psychological Association; 2004.

38- Pargament KI, Echemendia RJ, Johnson S, Cook P, McGath C, Mayers JG, et al. The conservative church: Psychological advantages avd disadvantages. Am J Community Psychol. 1987;15(3):269-86.

39- Mohammadi M. Resilience factors in individuals at risk for substance abuse [Dissertation]. Tehran: University of Social Welfare and Rehabilitation Sciences; 2005. [Persian]

40- Samani S, Jokar B, Sahragard N. Effects of resilience on mental health and life satisfaction. Iran J Psychiatry Clin Psychol. 2007;13(3):290-5. [Persian]

41- Snyder CR, Shorey HS, Cheavens J, Kimberly Mann P, Virgil H, Cynthia W. Hope and academic success in college. J Educ psychol. 2002;94(4):820-6.

42- Alexander ES, Onwuegbuzie AJ. Academic procrastination and the role of hope as a coping strategy. Pers Individ Differ. 2007;42(7):1301-10.

43- Kermani Z, Khodapanahi MK, Heydari M. Psychometric features of Omid Snyder's scale. Q J Appl Psychol. 2011;5(3):7-23. [Persian]

44- Nasiri H, Jokar B. The relationship between life's meaningfulness, hope, happiness, life satisfaction and depression. Women Dev Politics. 2008:6(2):157-76. [Persian]

45- Leoni JJ. A facilitator's manual for use with a spirituality -oriented group intervention protocol in combat veterans with chronic combat-related posttraumatic stress disorder [Dissertation]. Malibu, California: Pepperdine University; 2005.

46- Bagheri Zanjani L, Entesar Foumany G. The effectiveness of group based hope- therapy on increasing resilience and hope in life expectancy in patients with breast cancer. J Health Promot Manag. 2016;5(4):56-62. [Persian]
2013;12(2):146-59.

13- Alfred GC, Hammer JH, Good GE. Male student veterans: Hardiness, psychological well-being, and masculine norms. Psychol Men Masculinity. 2014;15(1):95-9.

14- Neff KD. The self-compassion scale is a valid and theoretically coherent measure of self-compassion. Mindfulness. 2016; 7(1): 264-74.

15- Denovan A, Macaskill A. Stress, resilience and leisure coping among university students: applying the broadenand-build theory. Leisure Stud. 2016;36(6):852-65.

16- Liu Y, Wang ZH, Li ZG. Affective mediators of the influence of neuroticism and resilience on life satisfaction. Pers Individ Differ. 2012;52(7):833-8.

17- Smith BW, Tooley EM, Montague EQ, Robinson AE, Cosper CJ, Mullins PG. The role of resilience and purpose in life in habituation to heat and cold pain. J Pain. 2009;10(5):493-500.

18- White B, Driver S, Warren AM. Resilience and indicators of adjustment during rehabilitation from a spinal cord injury. Rehabil psychol. 2010;55(1):23-32.

19- Mascaro N, Rosen DH. Assessment of existential meaning and its longitudinal relation with depressive symptoms. J soc clin psychol. 2008;27(6):576-99.

20- Kylma J. Dynamics of hope in adults living with HIV/AIDS: a substantive theory. J Adv Nurs. 2005;52(6):620-30.

21- Halama P, Dedova M. Meaning in life and hope as predictors of positive mental health: Do they explain residual variance not predicted by personality traits? Stud Psychol. 2007;49(3):191-200.

22- Shorey HS, Snyder CR, Yang X, Lewin Mr. The role of hope as a mediator in recollected parenting, adult attachment and mental health. J soc clin psychol. 2003;22(6):685-715.

23- Hasson-Ohayon I, Kravetz S, Meir T, Rozencwaig S. Insight into severe mental illness, hope, and quality of life on persons with schizophrenia and schizoaffective disorders. Psychiatry Res. 2009;167(3):231-8.

24- Johnson KL. The relationship of hope and quality of life in combat veterans seeking treatment for posttraumatic stress disorder. Kansas: University of Kansas, Psychology and Research in Education Publisher; 2001. pp. 29-58.

25- Kao YC, Liu YP, Chou MK, Cheng TH. Subjective quality of life in patients with chronic schizophrenia: relationships between psychosocial and clinical characteristics. Compr Psychiatry. 2011;52(2):171-80.

26- Ho WW, Chiu MY, Lo WT, Yiu MG. Recovery components as determinants of the health-related quality of life among patients with schizophrenia: structural equation modeling analysis. Aust $\mathrm{N} \mathrm{Z} \mathrm{J}$ Psychiatry. 2010;44(1):71-84.

27-Seligman MEP, Steen TA, Park N, Peterson C. Positive psychology progress: empirical validation of interventions. Am Psychol. 2005;60(5):410-21.

28- Snyder CR, Ilardi SS, Cheavens J, Michael ST, Yamhure L, Sempson S. The role of hope in cognitive behavior therapies. Cog Ther Res. 2000;24(6):747-62.

29- Charkhabi M, Mortazavi A, Alimohammadi S, Hayati D. The effect of spiritual intelligence training on the indicators of mental health in Iranian students: an experimental study. Procedia Soc Behav Sci. 2014;159:355-8.

30- Bahreinian A, Radmehr H, Mohammadi H, Bavadi B, 
49- Bronk KC, Hill PL, Lapsley DK, Talib TL, Finch H. Purpose, hope, and Life Satisfaction in three age groups. J Posit Psychol. 2009;4(6):500-10.

50- Ong AD, Edwards LM, Bergeman CS. Hope as a source of resilience in later adulthood. Pers Individ Differ. 2006;41(7):1263-73.

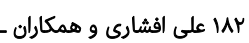

47- Dehghan F, Piri Kamrani M, Karami J. The effectiveness of group spiritual therapy on resilience. J Psychol Relig. 2016;8(4):81-92. [Persian]

48- McClure M. Use of Tai Chi to treat mental health disorders in veterans. [Dissertation]. Minneapolis: Walden University; 2017. 